ISSN: 1130-3743 - e-ISSN: 2386-5660

DOI: http://dx.doi.org/10.14201/teoredu301259272

\title{
EDUCACIÓN INFORMAL, VIDA COTIDIANA Y APRENDIZAJE TÁCITO
}

\section{Informal education, everyday life and tacit learning}

\section{Éducation informelle, vie quotidienne et aprentissage tacite}

José Luis RODRÍGUEZ ILLERA

Universitat de Barcelona, Facultad de Educación, Departamento de Teoría e Historia de la Educación. Pg. Vall d'Hebron, 171. 08035 Barcelona.

jlrodriguez@ub.edu

Fecha de recepción: enero de 2018

Fecha de aceptación: marzo de 2018

RESUMEN

Este artículo propone una revisión del concepto de educación informal, conectándolo con los contextos de actividad y aprendizaje que se dan en la vida cotidiana, y con el tipo de cuestiones a explicar que no aparecen de manera clara en la educación formal. Estas incluyen, entre otras, predisposiciones para comportarse en situaciones sociales, formas y tipos de decisiones, pertenencia y participación en comunidades, evolución de la identidad personal.

Se ha realizado una indagación bibliográfica y conceptual, en especial de autores que han explorado formas de aprendizaje poco consideradas en teoría de la educación, así como de los conceptos asociados de aprendizaje implícito, tácito, y de la idea de contextos de aprendizaje. Como resultado se propone una nueva clasificación de las formas de educación informal, así como las ventajas y problemas asociados a un enfoque basado en contextos de aprendizaje.

Palabras clave: contextos de aprendizaje; educación informal; vida cotidiana; aprendizaje tácito. 


\section{SUMMARY}

This article proposes a review of the concept of informal education, connecting it with the contexts of activity and learning that occur in everyday life, and the kinds of issues to explain that they do not appear clearly in formal education. These include, among others, predispositions to behave in social situations, forms and types of decisions, membership and participation in communities, development of personal identity.

It has been done a bibliographic and conceptual research, especially of authors who have explored ways of learning little considered in theory of education, as well as associated implicit, tacit learning concepts, and the idea of contexts of learning. As a result, it is proposed a new classification of the forms of informal education, as well as the advantages and problems associated with an approach based on learning contexts.

Key words: learning contexts; informal education; everyday life; tacit learning.

\section{SOMMAIRE}

Le présent document propose un examen du concept d'éducation informelle, connectant avec les contextes de l'activité et l'apprentissage qui se produisent dans la vie quotidienne et la nature des questions pour expliquer qu'ils n'apparaissent pas clairement dans l'éducation formelle. Ceux-ci comprennent, entre autres, les prédispositions à agir dans des situations sociales, les formes et les types de décisions, l'adhésion et la participation dans les communautés, le développement de l'identité personnelle.

Fait une enquête bibliographique et conceptuelle, surtout des auteurs qui ont exploré des moyens d'apprentissage peu considérée dans la théorie de l'éducation, aussi bien que les concepts associés de l'implicite, apprentissage tacite, et de l'idée de contextes d'apprentissage. Par conséquent, on propose une nouvelle classification des formes de l'éducation informelle, ainsi que les avantages et les problèmes associés à une approche basée sur les contextes d'apprentissage.

Mots clés: contextes d'apprentissage; éducation informelle; vie quotidienne; apprentissage tacite.

\section{INTRODUCCIÓN}

Este artículo pone en relación algunos conceptos que habitualmente se piensan por separado, todos ellos algo borrosos y polisémicos, definidos desde distintas tradiciones tanto teóricas como aplicadas: educación informal, vida cotidiana y aprendizaje tácito. Cualquiera de ellos es polémico, en especial el de educación informal y la tripartición de la que forma parte. Sin embargo, no se pretende ni una revisión exhaustiva de ninguno de ellos, ni un análisis de su alcance histórico, 
aunque se intenta explicitar una determinada conexión, la que relaciona una visión de "partes" (o subtipos, o modalidades si se puede hablar de ese modo) de la educación informal con la idea de vida cotidiana, y de ambas con las formas tácitas de aprendizaje y construcción del conocimiento que se dan en ambas.

Para ello, y dado que se intenta enunciar una propuesta de conexión entre conceptos, vamos a empezar en primer lugar por esos mismos conceptos de educación informal y de vida cotidiana, de manera mínima, pero mostrando sus dificultades principales. En segundo lugar, revisaremos algunos campos afines pero muy diferenciados con el campo pedagógico, si bien limitándonos a un autor principal para cada uno de ellos, y con la mirada en el tipo de preguntas, acciones y aprendizajes que se formulan. Se puede adelantar que todos ellos incluyen una forma de aprendizaje no consciente que se coordina con el explícito de manera constante y que se produce de manera concomitante al aprendizaje en cualquier tipo de situación o contexto, o de «macrocontexto» (educación formal, informal). Finalmente, se abordan las ventajas y problemas de un enfoque contextual como el que se sugiere, los tipos de aprendizaje que se dan en algunos subtipos principales de educación informal, la cuestión general del paso entre contextos, así como la necesidad de un amplio programa de investigación para mejorar la teoría subyacente y la descripción detallada de la forma que adoptan tanto en la escuela como en la vida cotidiana.

\section{REVISITANDO LA EDUCACIÓN INFORMAL Y LA VIDA COTIDIANA}

Hay un interés renovado por comprender bien los contextos (lugares, sitios, escenarios) en los que se produce el aprendizaje, no solo por los contextos institucionales, sino, sobre todo, por aquellos otros que se dan en otras agencias y contextos sociales como la familia, el trabajo o, en general, la «vida cotidiana» (Trilla, 1986; Hager, 2012; Livingstone, 2006; Vadeboncoeur, 2006). También, desde luego, por los contextos nuevos que se han creado en la sociedad digital. En conjunto, este interés parece estar relacionado con los profundos cambios sociales y educativos que han acontecido como consecuencia de los avances tecnológicos, y de los avatares económicos y sociales, acelerados desde la segunda mitad del siglo xx, es decir, con cambios en las formas de aprender, su evolución a lo largo de la vida personal, los sitios o/y contextos donde se produce el aprendizaje y su valoración, así como los cambios en las formas de enseñar y organizar la experiencia formativa. Este interés ha hecho surgir la idea de que es necesario comprender no solo los aprendizajes que se producen sino también su interrelación, lo que a veces se denomina su ecología, o integración, sus dependencias mutuas (Coll, 2013).

Los discursos psicológicos y pedagógicos han insistido, con razón, en la importancia del proceso de aprendizaje como explicativo de sus resultados. Pero, en líneas generales, también han insistido en centrarse en estos sobre todo como resultados escolares. En general, aunque desde luego no de manera exclusiva, el conocimiento escolar ha privilegiado un tipo de conocimiento y de resultados de 
aprendizaje centrados en aspectos o dominios fundamentalmente "cognitivos", que se asemejan a razonamientos formales o abstractos, articulados entre distintas áreas del conocimiento escolar, y que suelen ser de tipo conceptual o procedimental. Este conjunto de saberes está organizado casi siempre en relación a otros saberes, parecidos, pero más complejos, jerárquicamente más abstractos, que tienen que ser aprendidos en grados o cursos posteriores.

Aquello que queda fuera de este esquema de funcionamiento suele ser menos valorado y apenas entra en consideración (aunque hay poderosos mecanismos de compensación social extraeducativos: el triunfo en los deportes, negocios, artistas, etc.). Es lo que ocurre con el conocimiento "fuera de la escuela», el que se aprende en la familia, el trabajo, con los amigos, de manera incidental, en la vida cotidiana. Es evidente que se trata de conocimiento segmentado y más desconectado entre los distintos sitios de aprendizaje, sobre todo si se compara con el conocimiento escolar (Bernstein, 1999; Moss, 2001), que se ha convertido históricamente en el más importante y el que clasifica al resto de aprendizajes. Su segmentación quiere decir que lo que se ha aprendido en un determinado contexto está separado de lo que se aprende en otro. Claro está que la separación puede no ser completa cuando se trata de contenidos parecidos, o relacionados temáticamente, pero en general es inadecuado suponer que se trata de conocimientos conectados entre sí, articulados y organizados, que responden a un fin deliberado y programado.

Quizá sea la razón por la que el conocimiento escolar es más valorado socialmente (sin entrar a considerar el sesgo ideológico de tal valoración), es decir, por su forma organizada, articulada y conectada, que le da un grado elevado de cohesión interna, una manera común de afrontar problemas complejos o que requieren coordinación entre distintos saberes -en gran parte es el conocimiento científico el responsable de esta alta valoración-.

Sin considerar aquí las variaciones internas de la idea de conocimiento escolar, siempre basado en la escritura y en la abstracción, sí lo haremos con ese conglomerado de contextos y prácticas que se ha denominado «educación informal». Si ya las variaciones en torno a la escuela son enormes, ocurre mucho más en cuanto a lo informal: ¿qué relación tienen situaciones tan dispares como ver la televisión, jugar en la calle con los amigos, comer con la familia, aprender inglés en un curso de internet, navegar y buscar información, moverse por la ciudad, hablar por teléfono móvil, trabajar en una oficina, trabajar en el campo, participar en un foro o en un juego? En fin, la lista podría ser mucho mayor si tomáramos más situaciones, incluyéramos las "casuales» y no solo las organizadas, o simplemente intentásemos hacer una lista sistemática de la vida cotidiana. Efectivamente no hay mucho en común, no hay un mínimo común, como no sea su pertenencia a ese constructo de "vida cotidiana" al que nos hemos referido (Lefebvre, 1987). Pero la "vida cotidiana» tampoco es uniforme, ni mucho menos como se ve al revisar la historia de sus teorizaciones por Sheringham (2006), al contrario es uno de esos conceptos cuya descripción conlleva que está internamente dividido en nichos situacionales muy diversos (como los mencionados y otros muchos más), que funcionan con 
conductas, objetivos y organización diferentes, a veces contrapuestos ${ }^{1}$. Podríamos agrupar algunos de esos contextos principales: familia, trabajo, amigos y relaciones sociales estables, televisión, internet, ocio y videojuegos, viajes, lectura y escritura, relaciones interpersonales esporádicas.

Esta visión "ampliada» de la vida cotidiana sin más especificaciones es parecida a algunas ideas de educación informal, tan genéricas que apenas tienen interés. Falta una concepción que las unifique o, al revés, que muestre cómo sus divisiones internas responden a algo más que situaciones sociales que "Ocurren" o "están» -algo muy fenomenológico, pero nada explicativo-. En el caso de la educación informal, o del aprendizaje informal de manera más restrictiva, ha sido largamente identificado con el aprendizaje que ocurre en el lugar de trabajo, cuando se produce, por considerar que el trabajo es la actividad "principal» de la edad adulta. Es verdad que tampoco hoy se piensa de esa manera, ni incluso en los países que más han defendido ese enfoque -como el Reino Unido-, por más que siga siendo un vector principal de su análisis y sobre el que más se ha publicado (McLean y Wilson, 2009; Marsick y Watkins, 1990; Hager y Halliday, 2009; Le Clus, 2011).

Sin embargo, vida cotidiana y educación informal comparten aspectos centrales, en especial sobre las formas de aprendizaje que son predominantes en ambas, así como su mutua exclusividad durante milenios cuando no existía la escuela, ni antes de ella la escritura, o, cuando existiendo ambas, la mayor parte de la población aprendía al margen de ellas. La fuerza que una institución social específica ha adquirido parece hacernos olvidar el carácter primario de las otras formas educativas, así como el hecho de que siempre son, por definición, mucho más amplias que la escolar, y que, en realidad, la educación formal es, en gran medida, una preparación para el mundo del trabajo y la actividad adulta.

A diferencia de la forma especializada que supone la escuela y sus variantes (currículum explícito, agentes profesionales para enseñarlo, evaluación y sanción social de lo aprendido), lo que ocurre en situaciones de vida cotidiana -que solo son «informales» cuando las comparamos con la escuela- se basa en mecanismos de aprendizaje básicos y casi siempre «espontáneos»: imitación-mímesis, juego, soporte mediante andamiaje, participación progresiva en el grupo, casualidad, narrativa y casos, comunidades de práctica, etc.

1. La idea de "vida cotidiana" recorre el siglo xx en multitud de autores y tendencias como un constructo descriptivo de la experiencia directa: desde la freudiana Psicopatología de la vida cotidiana, de 1901, hasta la Crítica de la vida cotidiana de Henri Lefebvre, de 1947 a 1981; La presentación de la persona en la vida cotidiana de Erving Goffman, de 1959; La Invención de lo cotidiano de Michel de Certeau, de 1980, o incluso la obra colectiva Historia de la vida privada (ARIÉs, 1986), por nombrar algunas influyentes (además de Schultz, Heller, Lukaks, Pérec, entre otros). Una amplia revisión de temas y tendencias en BENNET y WATSON (eds., 2002) y en SHERINGHAM (2006) para una visión detallada de los pensadores franceses, también en Highmore (2002) para la corriente británica de la Mass Observation, y algunas concepciones de autores concretos en Wolf (1982). En el caso de la educación se ha contrapuesto a la educación escolar en los enfoques socioculturales, ya desde Vygotsky y de sus continuadores hasta la actualidad, y en los de tipo más etnográfico como LAVE (1988) o Rivière (1996). 
Algunos autores (Schugurensky, 2006) han señalado, al intentar describir el aprendizaje informal, que puede haber tres formas o modalidades bien diferenciadas: el aprendizaje autodirigido, el incidental y el que se produce por la socialización. Sin embargo, otros autores, como Marsick y Watkins (1990), diferencian entre el informal y el incidental como formas distintas, al menos en el trabajo. Y el aprendizaje autodirigido no siempre es pensado como informal (Popovic, 2012). El propio Schugurensky matiza estas formas al señalar cómo unas son conscientes (autodirigido) y otras no lo son, o casi nunca (incidental), o en pocas ocasiones. En esa misma línea se podría añadir el que se produce en el trabajo y, en especial, el que da en la vida cotidiana -si diferenciamos este último del proceso de socialización-.

En definitiva, y a pesar de su interés, las subdivisiones entre formas del aprendizaje informal son difíciles por ser poco claras, por remitir más allá de lo que se denomina proceso de socialización, o la vida cotidiana -en sus también complejas subdivisiones- al aprendizaje incidental o al autodirigido, ambos en los extremos de un espectro. Pero, además, el valor procesal de la idea de socialización no liga bien con la idea de vida cotidiana, que no parece tener un principio y un final -sino que más bien es un organizador del tiempo en forma de ciclos repetitivos-. Probablemente, el constructo de vida cotidiana es de naturaleza distinta al de educación o/y aprendizaje informal, el primero más inmediato, descriptivo y vivenciado, tipo emic, que se reconoce en parte por la experiencia directa, no mediada (Saitó, 2000), aunque este sea un rasgo insuficiente por sí solo; mientras que la educación informal parece más una construcción teórica externa, de la que el propio sujeto puede no ser consciente en algunos casos, como cuando acontece incidentalmente o en determinadas formas de su vida día a día (Rodríguez Illera y Kaechele, 2009).

\section{DOS TIPOS DE APRENDIZAJE Y UN RESULTADO}

Estas clasificaciones de la educación informal tienen el defecto común de suponer que el aprendizaje es el mismo en todas ellas. De alguna manera lo es, desde luego, pues una vez realizado queda en el acervo cognitivo y social de cada sujeto. Pero los contextos en los que se produce son tan diferentes, los mecanismos y los resultados tan opuestos, que difícilmente podemos considerarlos como formas parecidas o cercanas de una categoría superior que las englobe. Al contrario, si miramos los resultados de lo que se ha aprendido (a pesar de que no sepamos exactamente cómo ha sucedido), las descripciones suelen ser bastante coherentes aunque marcando esas diferencias. Por ejemplo, la noción de habitus de Bourdieu (1980), que rescata la idea de que actuamos de manera casi automática ante muchas situaciones y problemas; que nos comportamos con una predisposición a comprender lo que estamos viviendo y a actuar en consecuencia; y que hay un componente social, y de clase, en la forma como lo hacemos. El habitus nos devuelve los resultados del aprendizaje, escolar o informal, de la socialización si se quiere, como algo integrado en la vida cotidiana y en el trabajo, y no solo como 
una cuestión de clasificación de esos resultados o de una psicologización de los mismos. A pesar de que el habitus está claramente sesgado a colocar en el foco la práctica no reflexiva, la docta ignorancia (De Certeau, 1980, citando a Durkheim, p. 106: "ce qui est pratique pure sans théorie»), su tema y su escala de análisis son centrales para saber en realidad qué nos preguntamos, y focalizar la pregunta por la relación entre el aprendizaje y la educación informal, alejándola de ser el "pariente pobre» de la educación formal, su reverso no deseado.

No solo Bourdieu sino muchos otros autores han reconsiderado la idea de aprendizaje y han buscado una perspectiva más grupal y social (Illeris, 2006, para una revisión con varias teorías). Sin duda, el trabajo inaugural de Lave y Wenger (1991) para resituar su foco de análisis en lo que luego se ampliaría a comunidades de práctica por el propio Wenger. O, de manera más tradicional, las etnografías descriptivas (Delbos y Jorion, 1984), preguntándose por las formas de transmisión en contextos profesionales, y la de Faure (2000) centrada en el aprendizaje de la danza, entre otras. Todas ellas participan de esa idea de describir e interrogarse por el aprendizaje y sus resultados en una escala cercana a la vida cotidiana, en los mecanismos de transmisión de conocimientos en ambientes informales, siempre situados y mediados por el propio cuerpo, y en preguntarse por las formas culturales (p. e. la concepción sobre la danza que tienen los propios profesores, la pertenencia a un grupo profesional) que son aprendidas.

El concepto de habitus, y también este tipo de etnografías señaladas, incluyendo a interaccionistas al estilo de Goffman (1959), reintroducen una vieja idea, siempre mal resuelta, sobre lo que se aprende además de los contenidos. Se podría decir: lo que se aprende (conocimiento, en sentido amplio) y cómo se aprende. Se crea un conocimiento tácito que, en general, se ha aprendido tácitamente. Tácito es equivalente a no consciente, no verbalizado o, mejor, no explícito.

Ha sido Polanyi $(1958,1966)$ quien más ha insistido en la necesidad de un conocimiento tácito, tanto en la vida cotidiana como en la investigación científica. Polanyi da un ejemplo pregnante: sabemos cómo ir en bicicleta, pero no sabemos explicarlo, y si lo intentamos siguiendo unas instrucciones probablemente no lo lograremos. Es decir, "sabemos» cómo hacer determinadas acciones, pero no sabemos exactamente en qué consisten; sabemos más de lo que podemos explicar. Para él no hay nada de misterioso, ni de oculto, ni de esotérico, en esa "tacitidad", por el contrario, se trata de un mecanismo general de significación que está presente en todo conocimiento.

Polanyi cita en su apoyo una de las primeras publicaciones de Arthur Reber, un psicólogo cognitivo que ha dedicado todo su trabajo a estudiar lo que denomina aprendizaje implícito. El aprendizaje implícito es el que se da de manera "automática" y no intencional, de manera no consciente, mediante asociaciones principalmente, en situaciones cualesquiera y, en muchos casos, sin intervención de la voluntad, la atención o la concentración. Reber (1993) diseña una situación experimental en la que se presentan grupos de letras sin significado, en una secuencia determinada, sin conexión aparente; en una segunda secuencia, los sujetos son 
capaces de predecir partes siguientes de la secuencia como si hubieran aprendido la "gramática" que la genera, aunque no tienen ningún tipo de conciencia sobre la misma ni pueden verbalizarla. Esta manera de comprobar el aprendizaje ha sido ratificada por otros autores, y, a pesar de algunas críticas, se considera uno de los puntos fuertes del enfoque de Reber.

En un trabajo anterior (Reber, 1992) compara y toma alguna distancia entre su propio uso del aprendizaje tácito o implícito y el de Polanyi, si bien se trata de aproximaciones parecidas -quizá no tanto en el caso del Polanyi filósofo, aunque otros autores como Gascoigne y Thornton (2013) llevan a cabo una crítica filosófica del conocimiento tácito-. Dos tipos de aprendizaje, en general denominados explícito e implícito, este segundo una especie de inconsciente cognitivo en palabras de Reber o de Bargh (2017), contrapuestos por el grado de conciencia que se tiene sobre lo aprendido.

El aprendizaje implícito sucede, aunque no se busque, está de alguna manera sobredeterminado por el sensorium humano, y acontece por el simple hecho de estar en un contexto o situación y recibir sus estímulos. Es «incidental» más que fruto de la serendipia, de la ocasión continuada antes que de la excepcional. Reber lo sitúa como un mecanismo fundamental, anterior y más general que el aprendizaje explícito y que la educación, de carácter evolutivo y una vía diferente y separada del aprendizaje. La primacía del aprendizaje implícito, para Reber, viene de su carácter filogenético, anterior y más fundamental que el explícito. Otros autores, como Pozo (2014), quien, al revisar estos y otros enfoques, aboga por una integración de los dos tipos de aprendizaje en un sistema unificado, algo que parece lógico aunque se esté todavía muy lejos de lograrlo. García Carrasco (2015) propone situar la empatía y la imitación como bases de la educación informal, buscando explicar formas de aprendizaje en situaciones filogenéticas no intencionales y prelingüisticas, de alguna manera en línea con ideas del inconsciente cognitivo de Reber.

Además de Reber o de Bargh, también otros autores han intentado describir esta "doble vía» del aprendizaje y comportamiento humanos. Quizá el más conocido y sistemático sea Kahneman (2011), quien ha demostrado la existencia de dos sistemas en la toma de decisiones, uno rápido, casi instantáneo, por el que tomamos decisiones inmediatas, sin pensar, de manera poco o nada consciente; otro lento, muy consciente y premeditado, reflexivo y en el que valoramos distintos cursos de acción. El primero lo denomina S1, y está encargado de las decisiones que no pueden posponerse, o que se han automatizado y convertido en muchos casos en "espontáneas». El segundo lo denomina S2, y entra en funcionamiento casi siempre que hay una deliberación o una decisión que se considera compleja. En fin, S1 y S2 están combinados entre sí. Kahneman recurre también al aprendizaje primado (priming) para comprender cómo algunos estímulos presentes en la situación, verbales o no, desencadenan preferencias cuando son percibidos, aunque sea sin prestarles atención consciente. El primado es algo bien conocido en las teorías del aprendizaje, si bien tiene un interés algo limitado por no poseer un alto valor 
"ecológico" para la educación pues busca los mecanismos no conscientes por los que el sistema (perceptivo) crea los lazos según determinadas características de los estímulos (p. e. primado, secuencia) y genera un tipo de aprendizaje de bajo nivel, aunque pueda ser de una gran complejidad (Förster et al., 2009).

Esta breve revisión nos ha mostrado cómo distintos autores, de campos muy diversos, no siempre centrados en el aprendizaje o en la educación, proponen concepciones alternativas y se esfuerzan por señalar esa doble vertiente de la acción humana. No están hablando de los mismos fenómenos por lo que no se puede considerar que todo lo anterior sea coherente ni que "resuelvan" ningún tipo de cuestión común. Su valor reside en que plantean un problema pregnante (cómo tomamos decisiones, la transmisión cultural en ausencia de la escuela, formas de reaccionar ante situaciones, etc.), es decir, un problema que es percibido como importante por los propios agentes de la actividad, no solo como algo meramente formal o académico. Muchos de estos fenómenos han pasado a ser "automáticos", formando parte del gran depósito de conocimiento tácito, a su vez adquirido de manera implícita y tácita por el simple hecho de "estar ahí», pero no solo como un inconsciente cognitivo y personal sino como algo claramente social y cultural -sin intentar contraponer "cognitivo" $\mathrm{y}$ "Social»-, es decir, organizado de manera compleja, como una estructura, que es compartido públicamente (Collins, 2010, para tipos diferentes de conocimiento tácito).

En resumen, se aprende de manera explícita e implícita simultáneamente, tanto en la escuela como en la vida cotidiana en general, siempre en contextos de actividad y aprendizaje, con un resultado de aprendizaje integrado en formas de conocimiento con muy distinta consideración social, que además contienen formas tácitas incorporadas. Una parte de esos aprendizajes se realizan de manera "no consciente" y solo son verbalizables con dificultad, o no son verbalizables en absoluto, pero se unen a los explícitos y pasan a conformar el habitus, los esquemas cognitivos y sociales de percepción y acción, que actúan de manera automática o casi automática.

\section{VENTAJAS y CUESTIONES ABIERTAS}

Recuperar los procesos implícitos y tácitos, las formas tácitas del conocimiento educativo y del conocimiento en general, así como el lugar primero de la vida cotidiana (de lo social, en términos amplios), y los contextos especializados de aprendizaje, escolares e informales, son algunos de los ejes de reflexión para repensar la educación informal o, mejor dicho, la conceptualización clásica de la educación formal e informal y las relaciones entre ambas. Aunque sea excesivamente ambicioso, el proyecto marca un horizonte, lleno de ventajas y de problemas, que quisiéramos discutir.

Algunas de sus ventajas teóricas ya han sido mencionadas: la principal es que cuestiona una división muy simple, y simplista, de la totalidad educativa. No todo es tan evidente como esa división sugiere, ni lo formal y lo informal tienen una 
existencia separada más allá de su conceptualización, si bien conforman procesos, contenidos y objetivos de aprendizaje muy diferenciados. Al contrario, la propuesta es comprender mejor esos contextos de actividad y aprendizaje, como una alternativa a la diferenciación entre educación formal e informal y a las subdivisiones que «lo informal» ha recibido.

La educación informal ha sido pensada de muchas maneras, pero las más generales incluyen, como hemos visto, contextos tan diferentes como el autoaprendizaje y los acontecimientos casuales. Por ello mismo, creemos que hay que diferenciarlos y no denominarlos con referencia a un supuesto concepto organizador común. El siguiente cuadro sintetiza esos contextos principales que se suelen adscribir a la educación informal, a la vez que muestra sus diferencias profundas en función de los tipos de aprendizaje que están implicados de manera prioritaria.

\section{CUADRO 1}

CLASIFICACIÓN DE LA EDUCACIÓN INFORMAL

\begin{tabular}{|c|c|c|}
\hline CONTEXTOS & APRENDIZAJES & MODALIDAD PRINCIPAL \\
\hline $\begin{array}{c}\text { Contextos vinculados } \\
\text { al aprendizaje }\end{array}$ & $\begin{array}{c}\text { Aprendizaje autodirigido, } \\
\text { instrucción }\end{array}$ & Explícito \\
\hline No vinculados («estar ahí») & Incidental / espontáneo & Implícito, tácito \\
\hline $\begin{array}{c}\text { Híbridos: trabajo, } \\
\text { comunidades y } \\
\text { vida cotidiana }\end{array}$ & "Goal-based», serendipia \\
\hline
\end{tabular}

Elaboración propia.

Como ya hemos señalado, en todo contexto se producen aprendizajes explícitos e implícitos, aunque en distinto grado, por lo que cualquier clasificación siempre tiende a organizarlos de manera aproximada. Incluso si consideramos que el aprendizaje autodirigido pertenece al ámbito informal, y que es un contexto directamente organizado para "aprender" como objetivo último, dirigido conscientemente por el propio aprendiz que (auto)regula su aprendizaje, objetivos y ritmo. Otras formas de aprendizaje, no predecibles y en algunos casos no conscientes (p. e. priming, framing), acontecen en el interior de las situaciones instructivas más programadas y detalladas.

Una ventaja asociada a este enfoque "contextual» es pensar los contextos de actividad y aprendizaje como auténticas unidades de análisis para la educación. Si bien son unidades de escala pequeña o media (no como las de educación formal o informal), conectan las actividades y aprendizajes realizados con la forma que adoptan los resultados, con el conocimiento y competencias y su valoración tanto personal como social. Permiten además ser utilizadas tanto en educación formal como informal, constituyendo una alternativa a esas clasificaciones tan generales 
que ya hemos criticado, y uniéndose a otros enfoques no pedagógicos (psicología, antropología, sociología) que también toman los contextos como un concepto fundamental.

Hay un segundo grupo de cuestiones que aparece con esta «clasificación» de los contextos de actividad y aprendizaje, y que se refieren a cómo los distinguimos, de qué manera "pasamos" de uno a otro(s), así como comprender si pueden coexistir más de uno en un mismo espacio y tiempo. Dentro de todo ello existe una gran complejidad pues algunos son contradictorios, promueven formas de conocimiento en ocasiones opuestas, o poco o nada conectadas entre sí, pero otros parecen unidos de manera "transparente", sin costura, como si estuvieran profundamente coordinados. Esta es una problemática muy amplia, que ha adquirido fuerza en los últimos años bajo la forma del aprendizaje entre contextos (Vadeboncoeur [ed.], 2014; Bronkhorst y Akkerman, 2014; Erstad [ed.], 2016; Molas y Rodríguez Illera, 2018), es decir, por ejemplo, el paso de la escuela a la familia o, con más imprecisión, de situaciones formales a informales o a la inversa.

Claro está que estas cuestiones enunciadas requieren un desarrollo propio, que, según creemos, debe de ser situado a distintos niveles o escalas de análisis. Por ejemplo, en otro lugar (Rodríguez Illera, 2015) hemos sostenido que los contextos de actividad y aprendizaje pueden ser pensados con tres características comunes a todos ellos, aunque muy generales: unos marcadores de contexto que nos permiten distinguir ante qué tipo de contexto (o de actividad) estamos, un foco que es la parte visible de la actividad que se realiza (p. e. enseñar y aprender, pero también jugar, pasear, leer, etc.) y que normalmente se ha considerado como lo único que se debía comprender o sobre lo que se debía intervenir, y también un trasfondo que es el conjunto de supuestos (personales, culturales, históricos) sobre los que se organiza la actividad. Es evidente que estas distinciones están realizadas de manera abstracta y que tendrían que completarse con análisis de detalle de cada característica.

Por tanto, la conexión entre un enfoque "contextual» dentro de la teoría de la educación y las formas de los aprendizajes realizados, así como el carácter tácito o no de los mismos, tiene por delante un largo camino. Quizá el haber intentado resituar algunas ideas sobre la educación informal y su conexión con la vida cotidiana sea un primer paso en una reflexión que solo se inicia.

\section{CONClusiones}

No podemos concluir con hallazgos definitivos que amparen el recorrido que hemos hecho: solo podemos tomar nota de las vías que abre y de los problemas, teóricos y aplicados, que se plantean. La denominada "educación informal» es un claro desafío que está lejos de resolverse, quizá por el énfasis casi exclusivo puesto en la educación formal durante muchas décadas.

Sin embargo, hay indicadores claros de que se están empezando a dedicar muchos recursos al tema de la educación informal (Cuadrado, 2017), bajo formas 
diversas: aprendizaje en el trabajo, formas no escolares de educación, estudio de contextos informales como la familia, los medios o el aprendizaje incidental, así como el diseño de situaciones informales. Y también de recursos teóricos: una aproximación a las innovaciones y conceptos en disciplinas tradicionales no pedagógicas (como Sociología, Antropología, Psicología, e incluso Filosofía y Economía), o nuevos enfoques como la Teoría de la Actividad, o aproximaciones narrativas y contextuales, entre otros.

Muchas de estas ideas, unas nuevas y otras no, nos han llevado a intentar relacionar la educación informal con la vida cotidiana. Otro término que esconde su propia polisemia y dificultad, pero que tiene la ventaja de ser más intuitivo y sobre el que existe un gran número de aproximaciones. La vida cotidiana recubre multitud de contextos más acotados, más mesocontextos si se quiere, pero que no siempre son coherentes entre sí -al menos en cuanto a los resultados de los aprendizajes que se producen en ellos ni tampoco de la forma y conexión que adopta el conocimiento producido-. Pero tiene la ventaja de reintroducir como importante un campo muchas veces descuidado, así como el tipo de problemas que otras disciplinas consideran central. Hay muchas conexiones entre la educación informal y la vida cotidiana, aunque ninguno de los dos conceptos recubra al otro, y hemos querido explorar un tipo de aprendizaje que se produce en ambas: el aprendizaje implícito, a veces llamado tácito, que no se ciñe a los "contenidos" sino a aspectos no conscientes del proceso de aprendizaje.

Esto nos ha permitido una clasificación de los principales contextos y modalidades de aprendizaje que acontecen en la educación informal, así como plantear algunas de las ventajas y problemas del enfoque contextual (el aprendizaje entre contextos, o la estructura común a los contextos de aprendizaje formales e informales). Algo que consideramos un primer paso para seguir avanzando.

\section{REFERENCIAS BIBLIOGRÁFICAS}

ARIÈs, P. (1986) Pour une histoire de la vie privée, en ARIÈs, P. y DuBY, G. (eds.) Histoire de la vie privée, vol. III. Paris, Seuil, 7-19.

BARGH, J. A. (2017) Before you Know it. The unconscious reasons we do what we do. New York, Touchstone.

Bekerman, Z.; Burbules, N. y Silberman-Keller, D. (2006, eds.) Learning in places. The Informal Education Reader. New York, Peter Lang.

Bennet, T. y Watson, D. (2002) Understanding everyday life. Open University Press.

BernsteIn, B. (1999) Vertical and Horizontal Discourse: an essay. British Journal of Sociology of Education, 20, 2, 157-173.

Bourdieu, P. (1980) Le sens pratique. Paris, Minuit.

Bronkhorst, L. H. y AKKerman, S. F. (2016) At the boundary of school: Continuity and discontinuity in learning across contexts. Educational Research Review, 19, November 2016, 18-35. 
Coll, C. (2013) La educación formal en la nueva ecología del aprendizaje: tendencias, retos y agenda de investigación. En RODRíguez Illera, J. L. (comp.) Educación y aprendizaje en la sociedad digital. Barcelona, Universitat de Barcelona, 156-170.

Collins, H. (2010) Tacit and Explicit Knowledge. Chicago, University of Chicago Press.

CUAdRAdo Esclapez, T. (2017) La enseñanza que no se ve. Educación informal en el siglo XXI. Madrid, Narcea.

De Certeau, M. (1980) L'invention du quotidien. 1. Arts de faire. Paris, Gallimard, 1990.

Dejours, C. (1993) Intelligence pratique et sagesse pratique. Éducation permanente, 116, $47-70$

Delbos, G. y Jorion, P. (1984) La transmission des savoirs. Paris, Maison des Sciences de l'homme.

Erstad, O.; Kumpulainen, K.; Mäkitalo, A.; Schrøder, K. C.; Pruulmann-Vengerfeldt, P. y JóHANNSDÓtTiR, T. (eds.) (2016) Learning across contexts in the knowledge society. Rotterdam, NL: Sense Publishers.

Faure, S. (2000) Apprende par corps. Paris, La Dispute.

Förster, J.; Liberman, N. y Friedman, R. S. (2009) What Do We Prime? On Distinguishing Between Semantic Priming, Procedural Priming, and Goal Priming. En Morsella, E. et al. (eds.) Oxford Handbook of Human Action, 173-195.

García CARRASCO, J. (2015) La Teoría de la Educación y los mecanismos neuronales de la empatía. Deliberación sobre el escenario informal de educación. Temps d'Educació, 49, 23-47.

Gascoigne, N. y Thornton, T. (2013) Tacit Knowledge. Durham (uk), Acumen Publishing.

Goffman, E. (1959) La presentación de la persona en la vida cotidiana. Buenos Aires, Amorrortu.

Hager, P. (2012) Informal learning: everyday living. En The Routledge international handbook of learning. Oxon, Rouhagtledge, 207-215.

Hager, P. y Halliday, J. (2009) Recovering Informal Learning. Dordrecht, Springer.

Highmore, B. (2002) Everyday Life and Cultural Theory. London, Routledge.

ILLERIS, K. (2006) How We Learn: Learning and Non-Learning in School and Beyond. London, Routledge.

Jarvis, P. y WatTS, M. (eds.) (2012) The Routledge international handbook of learning. Oxon, Routledge.

Kahneman, D. (2011) Thinking, Fast and Slow. London, Penguin Books.

Kozulin, A. et al. (eds.) (2003) Vygotsky's educational theory in cultural context. New York, Cambridge University Press.

LAVE, J. (1988) Cognition in Practice; mind, mathematics and culture in everyday life. Cambridge, Cambridge University Press. [ver. cast. La cognición en la práctica. Barcelona, Paidós].

Lave, J. y Wenger, E. (1991) Situated learning: Legitimate peripheral participation. Cambridge, Cambridge University Press.

Lefebvre, H. (1987) The Everyday and Everydayness. Yale French Studies, 73, 7-11.

Le Cuus, M. A. (2011) Informal learning in the workplace: A review of the literature. Australian Journal of Adult Learning, 51 (2), 355-373.

Livingstone, D. W. (2006) Informal Learning: Conceptual Distinctions and Preliminary Findings. En BEKERMAN et al. (eds.) Learning in places. The Informal Education Reader. New York, Peter Lang, 203-228. 
MaClEAN, R. y WiLSON, D. (eds.) (2009) International Handbook on Education for the changing World of Work. New York, Springer.

Marsick, V. y WatKINS, K. (1990) Informal and incidental learning in the workplace. London, Routledge.

Molas Castells, N. y Rodríguez Illera, J. L. (2018) Activity and learning contexts in educational transmedia. Digital Education Review, 33.

Morsella, E.; Bargh, J. A. y Gollwitzer, P. M. (eds.) (2009) Oxford Handbook of Human Action. Oxford, Oxford University Press.

Moss, G. (2001) On Literacy and the Social Organisation of Knowledge Inside and Outside School. Language and Education, 15, 2-3, 146-161.

Polanyi, M. (1958) Personal Knowledge: Towards a Post-Critical Philosophy. Chicago, University of Chicago Press, 1974.

Polany, M. (1966) Tacit Dimension. Gloucester, Peter Smith, 1983.

Popovic, K. (2012) Self-directed learning. En The Routledge international handbook of learning. Oxon, Routledge, 216-227.

Pozo, J. I. (2014) Psicología del aprendizaje humano. Madrid, Morata.

Reber, A. S. (1992) Personal knowledge and the cognitive unconscious. Polanyiana, 3, $97-115$.

Reber, A. S. (1993) Implicit Learning and Tacit Knowledge. An Essay on the Cognitive Unconscious. New York, Oxford University Press.

RivièRe, C. (1996) Pour une théorie du quotidien ritualisé. En La Ritualisation du quotidien, Ethnologie française, 26, 229-238.

Rodríguez Illera, J. L. (2015) Los contextos y sus aprendizajes. Temps d'Educació, 48, 287-304.

Rodríguez IllerA, J. L. y KAECHELE, M. (2009) Digital Literacy and Activity Systems in Adolescents, en ZAJDA, J. y GiBBS, D. (eds.) Comparative Information Technology Languages, Societies and the Internet. New York, Springer Verlag, 89-102.

SAITó, T. (2011) Beautiful Fighting Girl. Minneapolis, University of Minnesota Press.

Schugurensky, D. (2006) "This Is Our School of Citizenship»: Informal Learning in Local Democracy. En BEKERMAN et al. (eds.) Learning in places. The Informal Education Reader. New York, Peter Lang, 163-182.

Sheringam, M. (2006) Everyday Life. Theories and Practices from Surrealism to the Present. Oxford, Oxford University Press.

TriLla, J. (1986) La educación informal. Barcelona, PPU.

VAdeboncoeur, J. (2006) Engaging Young People: Learning in Informal Contexts, en Green, J. y Luke, A. (eds.) Rethinking Learning: What Counts as Learning and What Learning Counts. Washington DC, AERA, 239-278.

VAdeboncoeur, J. (ed.) (2014) Learning in and across contexts. Reimagining Education. New York, Teachers College University of Columbia.

Wolf, M. (1982) Sociologías de la vida cotidiana. Madrid, Cátedra. 